\title{
EXTENDING CLOSED PLANE CURVES TO IMMERSIONS OF THE DISK WITH $n$ HANDLES
}

BY

\author{
KEITH D. BAILEY(1)
}

\begin{abstract}
Let $f: S \rightarrow E$ be a normal curve in the plane. The extensions of $f$ to immersions of the disk with $n$ handles $\left(T_{n}\right)$ can be determined as follows. A word for $f$ is constructed using the definitions of Blank and Marx and a combinatorial structure, called a $T_{n}$-assemblage, is defined for such words. There is an immersion extending $f$ to $T_{n}$ iff the tangent winding number of $f$ is $1-2 n$ and $f$ has a $T_{n}$-assemblage.

For each $n$, a canonical curve $f_{n}$ with a topologically unique extension to $T_{n}$ is described $\left(f_{0}=\right.$ Jordan curve). Any extendible curve with the minimum number $(2 n+2$ for $n>0)$ of self-intersections is equivalent to $f_{n}$.
\end{abstract}

Introduction. Let $f: \partial M \rightarrow N$ be a regular map ( $C^{1}$-immersion) of the boundary of a compact, oriented surface into an oriented surface without boundary. Since $M$ and $N$ admit the structure of Riemann surfaces, the problem of the existence and topological classification of holomorphic maps $F: M \rightarrow N$ that extend $f, F \mid \partial M=f$, have been of interest to many mathematicians, notably M. Morse and M. Heins [14], [15]. According to Stoillow-Whyburn theory [16], [22], a continuous map $F$ that is light, open, and sense-preserving on $M$ $-\partial M$ and a local homeomorphism relative to $M$ on $\partial M$ is topologically equivalent to a holomorphic mapping from $M$ to $N$. Such maps are now said to be properly interior. If the map is branch point free (local homeomorphism) and $C^{\mathbf{1}}$ on the boundary, then it is equivalent to a $\boldsymbol{C}^{\mathbf{1}}$-immersion [J. Jewett, 9]; [J. H. C. Whitehead, 20]. Accordingly, this study will be presented entirely in the $C^{\mathbf{1}}$. context.

Particular success has been obtained when the immersion $f$ has its oriented image [f] in the plane $E$ or sphere $S^{2}$ lying in completely general position (normal immersion); that is, $[f]$ has a finite number of transverse self-intersections (nodes). Such curves comprise a dense-open subset of $C^{1}(\partial M, N)$ in the $C^{1}$-topology [Whitney, 21] , and were combinatorily classified by Titus [17] by means of their Whitney-Titus intersection sequence. In contrast to arbitrary

Presented to the Society, April 28, 1973; received by the editors June 27, 1973 and, in revised form, March 15, 1974

AMS (MOS) subject classifications (1970). Primary 57D35.

(1) This paper is part of the author's Ph.D. dissertation written at the University of Illinois under Professor George K. Francis. 
immersions (presenting self-tangencies, multiple crossings and multiply covered arcs, etc.), the bounding properties of normal immersions are unaffected by sufficiently small deformations.

When the domain $\partial M$ of $f$ is the circle $S$ and $N$ is the plane, $E$, the tangent winding number $\tau(f)$ is the degree of the unit tangent vector. By the Whitney-Graustein theorem, $\tau(f)$ classifies $f$ up to regular homotopy. If $\partial M$ has $d$ components and $N$ is $S^{2}$ with base point $\infty$, then define $\tau(f)$ to be the sum of the tangent winding numbers computed in $E=S^{2}-\{\infty\}$. The character of extensions $F$ of $f$ has been found to depend on $\tau(f)$, the genus $n$ of $M$, the number $d$ of components of $\partial M$, and, if $F$ is properly interior, on a nonnegative integer $u$ associated with the multiplicities of the branch points. Finally, letting $b$ be the number of preimages of the spherical base point, Francis [5] found the constraint:

$$
\tau=2-d-2 n+u-2 b .
$$

The question of necessary and sufficient conditions for properly interior extensions to the disk of a normal, plane immersion of its boundary was posed by Loewner and resolved by Titus [18] by means of algorithms on the intersection sequence. Using novel invariants, called words, S. J. Blank [1] showed that for a given word for $f$, equivalence classes of immersions which extend $f$ are in oneto-one correspondence with the combinatorial structures on the word, called groupings. Such structures were called assemblages by M. Marx [12], who obtained a classification for properly interior maps of the disc in the plane. Francis [5] obtained the assemblages for spherical immersions of the disk and S. Troyer [19] the assemblages for plane immersions of a disk with $n$-holes.

Sufficient conditions, based on Titus' original methods, for the existence of properly interior extensions of plane curves to the annulus [Marx, 10], the torus minus a disk [Marx, 11] and spherical curves to the disk [Marx and Verhey, 13] are known.

This paper is concerned with determining extensions when $M$ has genus greater than zero. Let $T_{n}$ be a closed disk with $n$ handles. That is, $T_{n}$ is a closed, oriented surface of genus $n$ with an open disk removed. The purpose of this study is to determine the nature of $T_{n}$-assemblages for immersions when $N$ is the plane.

The correspondence between assemblages and extensions as well as the relation between genus and tangent winding number can be described as follows.

There is an immersion $F: T_{n} \rightarrow E$ such that $F \mid \partial T_{n}=f$ if and only if $\tau(f)=$ $1-2 n$ and $f$ has a $T_{n}$-assemblage.

Two immersions $F, G: T_{n} \rightarrow E$ are equivalent if there is an orientation preserving diffeomorphism $h: T_{n} \rightarrow T_{n}$ such that $F=G \circ h$. The equivalence 
classes of extensions of a loop $f$ and assemblages for $f$ are in one-to-one correspondence.

Some consequences of these results will be proved in the last section of this paper. The structure of a $T_{1}$-assemblage will be described without reference to the reflection operation and this form will be used to analyze a normal curve for which every node separates the curve into two nonempty disjoint pieces. In contrast to such curves bounding plane immersed discs [Francis, 3] this curve has three inequivalent extensions to $T_{1}$. A theorem of Marx [12] extends to arbitrary genus: If $f$ has a $T_{n}$-assemblage, then it has a properly interior extension to $T_{n}$.

Finally, a canonical class of normal curves is constructed. For each $n$, there is exactly one normal curve $f_{n}$ that has the fewest self-intersections and extends to an immersion of $T_{n}$. Moreover, the curve $f_{n}$ and its extension $F_{n}$ are topologically unique.

The author is pleased to express his appreciation to Professor George K. Francis of the University of Illinois, who suggested this thesis and whose generous contributions of ideas and time made its completion possible.

1. Preliminaries. Definitions and terminology needed to describe $T_{n}$ assemblages and state the main theorems will be presented in this section. The objects involved in this study are intervals, circles, the oriented Euclidean plane and compact, oriented surfaces. All mappings are taken to be sense-preserving $C^{1}$ immersions.

Let $h$ be a $C^{1}$-immersion of a closed interval or a circle into the plane $E$. If $h^{-1}(h(x))=\{x, y\}$ and the tangent vectors $h^{\prime}(x)$ and $h^{\prime}(y)$ are independent, then $h(x)$ is a node of $h$. If the only self-intersections of $h$ are nodes, then $h$ is normal and when the domain of $h$ is a circle, $h$ is a normal loop. Because nodes must be isolated and the domains used are compact, a normal map has only a finite number of nodes. Two normal maps $h$ and $g$ are transverse if $p=h(x)=g(y)$ implies that the tangent vectors $h^{\prime}(x)$ and $g^{\prime}(y)$ are independent and $p$ is not a node of $h$ or $g$. The image of a map $h$ will be denoted by $[h]$. If the interval $[x, y]$ is contained in the domain of $h$ and $a=$ $h(x)$ and $b=h(y)$ are not nodes of $h$, then the sense-preserving map $h \mid[x, y]$ can be unambiguously denoted by $(a, b) h$.

For each nonnegative integer $n$, let $T_{n}$ denote a closed disk with $n$ handles. Thus $T_{n}$ is a compact, oriented, connected surface of genus $n$ with an open disk removed. Let $S$ be the boundary of $T_{n}\left(S=\partial T_{n}\right)$ and orient $T_{n}$ so that if $S$ has the induced orientation, then the interior of $T_{n}$ is to the left of $S$.

Representations of $T_{n}$ which facilitate the study of their plane immersions can be obtained as follows. Attach opposite ends of $2 n$ rectangular strips 
to disjoint segments of the boundary of a closed disk so that the resulting space $X$ is smooth, orientable, and has only one boundary component. Then $X$ and $T_{n}$ are diffeomorphic. $T_{0}$ and $T_{1}$ have essentially one such representation, while $T_{n}$ may have several for $n \geqslant 2$. For each $n$, one representation can be obtained by attaching the ends of the strips to antipodal arcs of the boundary of of the disc. See Figure 1 for $T_{2}$.

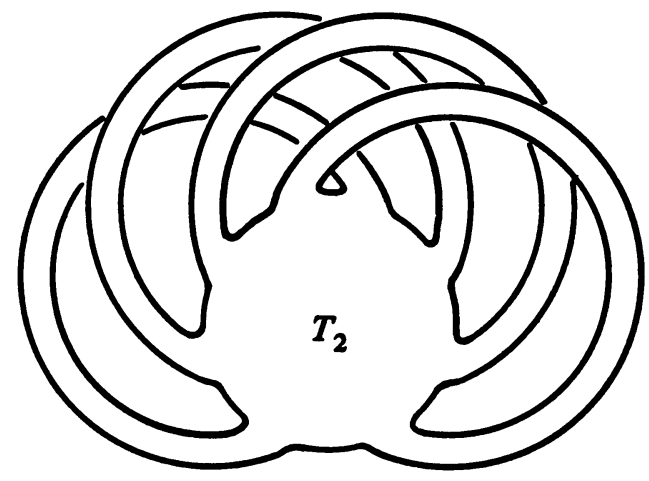

FIGURE 1

An illustration of the equivalence between adding a "handle" to a disc and adding a pair of "linked" strips to the boundary is given in Figure 2.
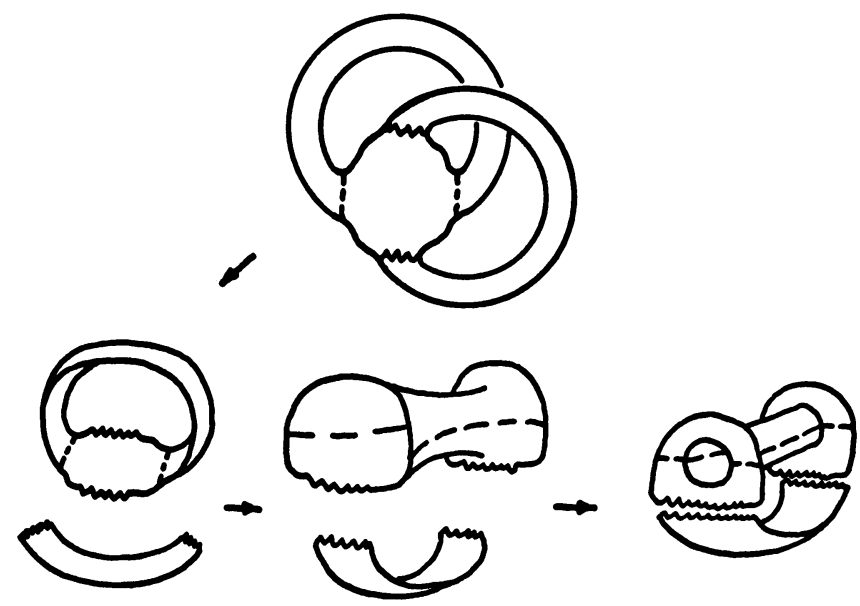

FIGURE 2

Let $f: S \rightarrow E$ be a normal loop. Then $E-[f]$ consists of a finite number of bounded components $\left\{E_{1}, \cdots, E_{m}\right\}$ and one unbounded component 
$E_{\infty}$. A raying $R$ for $f$ is a finite set of embeddings of the interval $[0,1]$, called rays, such that

(1) the oriented image of each ray begins in a bounded component of $E-$ [f] and ends in $E_{\infty}$;

(2) at least one ray begins in each bounded component;

(3) each ray and $f$ are transverse;

(4) the images of the rays are disjoint.

The direction in which rays cross [f], the relative order in which crossings occur on $[f]$, and the order of crossings on each ray (as well as the tangent winding number of $f$ ) comprise the essential data on $f$ used in this work to study extensions of $f$ to plane immersions of $T_{n}$. Let $R$ be a raying for $f$ and let $\alpha \in R$. If $\alpha(t)=f(x)=p$ is a crossing (intersection), the sign of $p$ is defined by $\operatorname{sgn}(p)=\operatorname{sgn} \operatorname{det}\left[\alpha^{\prime}(t), f^{\prime}(t)\right]$. Thus $\operatorname{sgn}(p)=1$ if $\alpha$ crosses $[f]$ from left to right and $\operatorname{sgn}(p)=-1$ otherwise (see Figure 3 ). The position integer, $\operatorname{pos}(p)$, is defined so that $\operatorname{pos}(p)=k$ if $p$ is the $k$ th crossing on $\alpha$. A list of the crossings in the order they occur as $[f]$ is traced is called a crossing word $W(f, R)$ for $f$. Different starting points correspond to cyclic permutations of a given word and thus the circular order on crossings induced by $S$ is unaffected. As an ingredient in a word, a crossing, together with its sign and position integer, is also called a letter. The idea of using rays to generate words of signed crossings was first used by Blank [1]. Marx [12] extended Blank's results using words of signed crossings which also carried indices corresponding to the positions on the rays.

Let $\bar{p}$ and $p$ be crossings on a ray $\alpha$ such that $\operatorname{sgn}(\bar{p})=-1, \operatorname{sgn}(p)=$ 1 , and $\operatorname{pos}(\bar{p})<\operatorname{pos}(p)$. Then $P=(\bar{p}, p)$ is a pair from $W(f, R)$. Two pairs $P$ and $Q$ are linked if $f^{-1}(P)$ intersects both complementary components of $f^{-1}(Q)$ in $S$. Linked pairs can be easily recognized from the positions of the crossings in the word (see Figure 3). A disjoint collection $P$ of pairs which uses each negative crossing in $W(f, R)$ is called a pairing on $W(f, R)$.

For notational purposes, define the function $\Lambda: P \times P \rightarrow\{0,1\}$ by $\bigwedge(P, Q)=1$ if and only if $P$ links $Q(\wedge(P, P)=0)$. When more than one linking relation may be involved, $(P, \wedge)$ will denote the collection with relation determined by $\Lambda$. A subset $P^{\prime}$ of $P$ is properly nested if no two pairs in $P^{\prime}$ are linked-i.e., $\Lambda P^{\prime} \times P^{\prime}=0$.

Two pairs $P$ and $P^{\prime}$ in $P$ are similar with respect to $\wedge, P \wedge P^{\prime}$, if $\bigwedge(P, R)=\bigwedge\left(P^{\prime}, R\right)$ for each $R \in P\left(\right.$ in particular, $\left.\bigwedge\left(P, P^{\prime}\right)=0\right)$.

Examples of the above concepts are given in Figure 3. The subscript on a crossing is its position integer and a negative crossing is indicated by a bar over the letter. 

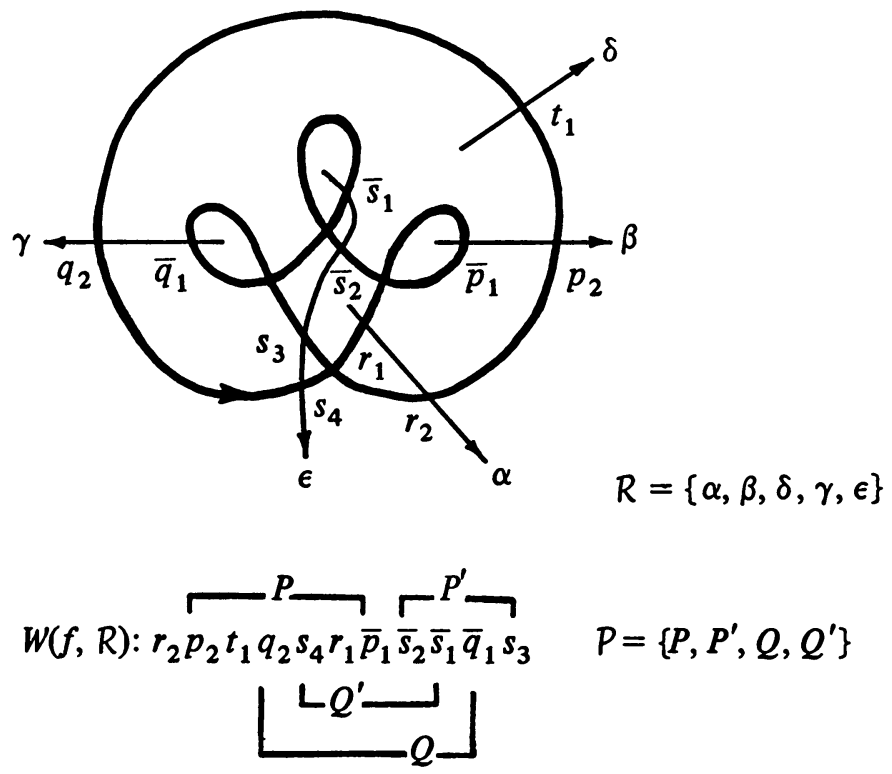

\begin{tabular}{l|l|l|l|l|l}
$\wedge$ & $P$ & $P^{\prime}$ & $Q$ & $Q^{\prime}$ & \\
\hline$P$ & 0 & 0 & 1 & 1 & \\
\hline$P^{\prime}$ & 0 & 0 & 1 & 1 & \\
\hline$Q$ & 1 & 1 & 0 & 0 & \\
\hline$Q^{\prime}$ & 1 & 1 & 0 & 0 &
\end{tabular}

$$
\begin{aligned}
& P \wedge P^{\prime} \\
& Q \wedge Q^{\prime}
\end{aligned}
$$

FIGURE 3

Assume that $P=(\bar{p}, p)$ and $Q=(\bar{q}, q)$ are linked pairs from $W(f, R)$. Then some cyclic permutation of $W(f, R)$ reads $A p B q C \bar{p} D \bar{q}$ or $A p B \bar{q} C \bar{p} D q$, where $A, B, C$, and $D$ are (possibly empty) subwords. In either case, the following definition is made. The reflection of $W(f, R)$ about $P$ and $Q$ is $W(P, Q)=A D C B$. The linking relation induced on $P_{0}=P-\{P, Q\}$ by the new arrangement of the letters will be denoted by $\Lambda_{1}$.

In $\S 3$, it will be shown that $\Lambda_{1}$ can also be derived from $\Lambda$. Although $W(P, Q)$ is not necessarily the crossing word for a normal loop, it will be shown in $\S \S 2$ and 3 to contain the essential information needed to study a normal loop obtained by modifying $f$. If $\left(P_{0}, \bigwedge_{1}\right)$ is not properly nested, then $P_{0}$ 
contains linked pairs about which $W(P, Q)$ can be reflected. If this process is continued, a (possibly empty) properly nested collection results. Suppose that $P_{1}, \cdots, P_{2 m}$ is a sequence in $P$ which can be used to carry out a succession of reflections; i.e., $W_{1}=W\left(P_{1}, P_{2}\right), W_{i}=W_{i-1}\left(P_{2 i-1}, P_{2 i}\right), \bigwedge_{i}$ is the relation on $P-\left\{P_{1}, \cdots, P_{2 i}\right\}$, and $\bigwedge_{i-1}\left(P_{2 i-1}, P_{2 i}\right)=1$ for $2 \leqslant i \leqslant m$. If $\left(P-\left\{P_{1}\right.\right.$, $\left.\left.\cdots, P_{2 m}\right\}, \wedge_{m}\right)$ is properly nested, then $C=\left(P_{1}, \cdots, P_{2 m}\right)$ is a decomposition sequence of length $2 \mathrm{~m}$. The principal definition in this work can now be stated.

1.1. Definition. Let $f: S \rightarrow E$ be a normal loop with raying $R$. Assume that $P$ is a pairing on $W(f, R)$ and $P$ contains a decomposition sequence of length $2 n$. Then $P$ is a $T_{n}$-assemblage.

A $T_{0}$-assemblage is therefore a properly nested pairing. An alternative description of $T_{1}$-assemblages which does not involve reflections will be given in $\S 7$.

The tangent winding number of a normal loop $f$ is defined by $\tau(f)=$ degree of $\left\{f^{\prime} /\left|f^{\prime}\right|: S \rightarrow S\right\}$.

The relationships among assemblages, $\tau(f)$, and immersions of $T_{n}$ which extend $f$ are given in the following two theorems.

1.2. TheOREM I. Let $f: S \rightarrow E$ be a normal loop. If $\tau(f)=1-2 n$ and a crossing word of $f$ has a $T_{n}$-assemblage, then $f$ can be extended to an immersion of $T_{n}$. Conversely, if $f$ can be extended to an immersion of $T_{n}$, then $\tau(f)=1-2 n$ and each crossing word of $f$ has a $T_{n}$-assemblage.

Let $F$ and $G: T_{n} \rightarrow E$ be immersions. Then $F$ and $G$ are equivalent if there is an orientation preserving diffeomorphism $h: T_{n} \rightarrow T_{n}$ such that $F=G \circ h$.

\subsection{TheOREM II. Let $f: S \rightarrow E$ be a normal loop and assume that $F$,} $G: T_{n} \rightarrow E$ are immersions which extend $f$. Then $F$ and $G$ are equivalent iff $F$ and $G$ determine the same $T_{n}$-assemblage on each crossing word of $f$.

Theorem II will be proved in $\S 6$. It will also be shown in $\S 6$ that the existence and equivalence of extensions of a normal loop depend only on the image curve and not on the parametrization. That these questions are independent of the copy of $T_{n}$ used will also follow.

2. Modifications. Let $f: S \rightarrow E$ be a normal loop with raying $R$. If $P$ and $Q$ are disjoint linked pairs from $W(f, R)$, a new normal loop $f_{1}$ can be defined using $P, Q$, and $f$. A raying $R_{1}$ can be obtained for $f_{1}$ by supplementing $R$ in such a way that the reflection $W(P, Q)$ of $W(f, R)$ is a subword of $W\left(f_{1}, R_{1}\right)$. Also, $\tau\left(f_{1}\right)$ will be calculated in terms of $\tau(f)$ and it 
will also be shown that for certain pairings the linking induced by $W\left(f_{1}, R_{1}\right)$ is determined by $W(P, Q)$.

A normal loop $f_{1}=\bmod (f, P, Q)$ will be constructed by deleting small intervals of $[f]$ which contain the crossings in $P$ and $Q$ and then reconnecting the result using arcs "parallel" to the segments of the rays determined by $\boldsymbol{P}$ and $Q$. Assume that $P=(\bar{p}, p)$ is on $\alpha$ and $Q=(\bar{q}, q)$ is on $\beta$ ( $\alpha$ and $\beta$ may be the same). Denote the segment $(\bar{p}, p) \alpha$ by $\alpha_{p}$ and $(\bar{q}, q) \beta$ by $\beta_{Q}$. Choose a simple arc $u_{L}$ to the left of $\alpha_{P}$ from $a$ to $b$ as in Figure 4 (some of the possible crossings of $f$ with $\alpha_{P}$ and $\beta_{Q}$ have been indicated). Assume that $u_{L}$ is sufficiently close to $\alpha$ so that it intersects $f$ in the same manner as does $\alpha_{P}$. Similarly, choose $u_{R}$ to the right of $\alpha$ from $b^{\prime}$ to $a^{\prime}$. Next choose $v_{L}$ and $v_{R}$, as indicated in Figure 4, to the left and right of $\beta$. In case $\alpha=\beta$, require that $u_{L}, u_{R}, v_{L}$, and $v_{R}$ are disjoint and $v_{L}$ and $v_{R}$ are "closer" to $\alpha$ than $u_{L}$ and $u_{R}$ (see, for example, Figure 5).

(a)

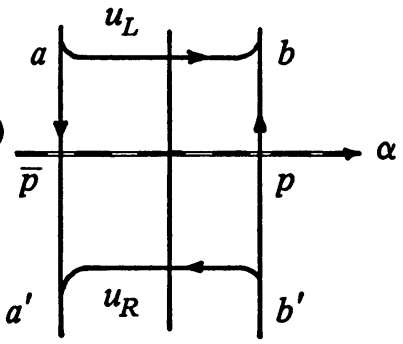

(b)

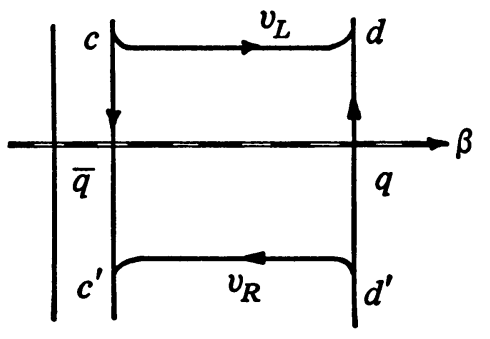

FIGURE 4

2.1. Definition. If the order is $p, \bar{q}, \bar{p}, q$ in $W(f, R)$, let

$$
\begin{aligned}
\bmod (f, P, Q)= & u_{L}+(b, c) f+v_{L}+\left(d, b^{\prime}\right) f \\
& +u_{R}+\left(a^{\prime}, d^{\prime}\right) f+v_{R}+\left(c^{\prime}, a\right) f
\end{aligned}
$$

if the order is $p, q, \bar{p}, \bar{q}$, let

$$
\begin{aligned}
\bmod (f, P, Q)= & u_{L}+\left(b, d^{\prime}\right) f+v_{R}+\left(c^{\prime}, b^{\prime}\right) f \\
& +u_{R}+\left(a^{\prime}, c\right) f+v_{L}+(d, a) f .
\end{aligned}
$$

\subsection{Proposition. $\tau \bmod (f, P, Q)=\tau(f)+2$.}

Proof. Abbreviate $\tau((x, y) f)$ to $\tau(x y)$ and assume (without loss of generality) that the order of the crossings is $p, \bar{q}, \bar{p}, q$. Let $f_{1}=\bmod (f, P, Q)$. Then (see Figure 4):

$$
\begin{aligned}
\tau(f) & =\tau(b c)+\tau\left(d b^{\prime}\right)+\tau\left(a^{\prime} d^{\prime}\right)+\tau\left(c^{\prime} a\right)+\tau\left(a a^{\prime}\right)+\tau\left(b^{\prime} b\right)+\tau\left(c c^{\prime}\right)+\tau\left(d^{\prime} d\right), \\
\tau\left(f_{1}\right) & =\tau\left(u_{L}\right)+\tau\left(u_{R}\right)+\tau\left(v_{R}\right)+\tau\left(v_{L}\right)+\tau(b c)+\tau\left(d b^{\prime}\right)+\tau\left(a^{\prime} d^{\prime}\right)+\tau\left(c^{\prime} a\right) .
\end{aligned}
$$

Thus 


$$
\begin{aligned}
\tau\left(f_{1}\right)-\tau(f)= & \left(\tau\left(u_{L}\right)-\tau\left(b^{\prime} b\right)+\tau\left(u_{R}\right)-\tau\left(a a^{\prime}\right)\right) \\
& +\left(\tau\left(v_{L}\right)-\tau\left(d^{\prime} d\right)+\tau\left(v_{R}\right)-\tau\left(c c^{\prime}\right)\right) .
\end{aligned}
$$

Since $u_{L}+\left(b^{\prime}, b\right)(-f)+u_{R}+\left(a, a^{\prime}\right)(-f)$ is a negatively oriented Jordan curve, $\tau=-1$ on this piecewise regular loop. The total contribution to $\tau$ on the corners is $(4)(-1 / 2)=$ -2 and therefore $\tau\left(u_{L}\right)-\tau\left(b^{\prime} b\right)+\tau\left(u_{R}\right)-\tau\left(a a^{\prime}\right)=1$. Similarly, $\tau\left(v_{L}\right)-\tau\left(d^{\prime} d\right)+$ $\tau\left(v_{R}\right)-\tau\left(c c^{\prime}\right)=1$. Thus $\tau\left(f_{1}\right)=\tau(f)+2$.

The relationship between rayings, pairings, and linking for $f$ and $f_{1}$ will be considered next. First note that if the crossings of $f_{1}$ on the rays in $R$ are listed in the order they are encountered as $f_{1}$ is traversed, the reflection $W(P, Q)$ of $W(f, R)$ is obtained. Thus the following proposition holds.

2.3. Proposition. The linking $\bigwedge_{1}$ on $P-\{P, Q\}$ induced by the reflection $W(P, Q)$ is the same as the linking induced by $\bmod (f, P, Q)$.

$W(P, Q)$ is not necessarily a crossing word for $f_{1}$ since new bounded components may be created by $u_{L}, u_{R}, v_{L}$, and $v_{R}$. Thus additional rays may be needed to obtain a raying $R_{1}$ and word $W\left(f_{1}, R_{1}\right)$ for $f_{1}$. Let $P_{0}=P-\{P, Q\}$. A pairing $P_{1} \supseteq P_{0}$ will be defined on $W\left(f_{1}, R_{1}\right)$ with linking relation $\Lambda_{1}$ induced by $f_{1}$. This relation restricted to $P_{0}$ is the same as the relation on $P_{0}$ induced by $W(P, Q)$. Moreover, each new pair in $P_{1}$ is similar to a pair in $P_{0}$. Thus all linking patterns are determined by $W(P, Q)$ and the new rays are essentially redundant. These statements are proved in the next proposition.

\subsection{Proposition. Let $(P, \wedge)$ be a pairing on $W(f, R)$ such that $P, Q \in P$} and $\Lambda(P, Q)=1$. A raying $R_{1} \supseteq R$ can be chosen for $f_{1}$ so that $W(P, Q)$ is a subword of $W\left(f_{1}, R_{1}\right)$ and a pairing $\left(P_{1}, \bigwedge_{1}\right)$ exists on $W\left(f_{1}, R_{1}\right)$ with the following properties. .

(1) $P_{0}=P-\{P, Q\} \subseteq P_{1}$.

(2) There is a projection $S: P_{1} \rightarrow P_{0}$ such that $S\left(R_{1}\right)$ and $R_{1}$ are similar, $S\left(R_{1}\right) \wedge_{1} R_{1}$, for each pair $R_{1} \in P_{1}$.

PRoof. Recall that $S\left(R_{1}\right) \bigwedge_{1} R_{1}$ means that $\bigwedge_{1}\left(S R_{1}, R\right)=\bigwedge_{1}\left(R_{1}, R\right)$ for each $R \in P_{1}$. A new ray is needed for each new component of $E-\left[f_{1}\right]$. Choose $\alpha_{1}, \cdots, \alpha_{m}$ to the left of $\alpha ; \beta_{1}, \cdots, \beta_{n}$ to the left of $\beta ; \alpha_{-1}, \cdots, \alpha_{-m^{\prime}}$ to the right of $\alpha$; and $\beta_{-1}, \cdots, \beta_{-n}$ ' to the right of $\beta$ (see Figure 5 for the case $\alpha=\beta$ ). Construct the rays parallel to and close enough to $\alpha$ and $\beta$ so that the new rays intersect $f$ in the same manner as do $\alpha$ and $\beta$. Then, except for initial positive crossings with $u_{L}, u_{R}, v_{L}$, and $v_{R}$, all new crossings occur as follows. Assume $\bar{p}_{0}$ is a negative crossing on $\alpha$ such that $\operatorname{pos}(\bar{p})<\operatorname{pos}\left(\bar{p}_{0}\right)$. Then there are new negative crossings $\bar{p}_{i}$ on $\alpha_{i}$ which are adjacent in the word $W\left(f_{1}, R_{1}\right)$ and occur as $\bar{p}_{m_{1}} \cdots \bar{p}_{1} \bar{p}_{0} \bar{p}_{-1} \cdots \bar{p}_{-m_{2}}$. If $p_{0}$ is a positive crossing on $\alpha$ beyond 
$\bar{p}$ (pos $\bar{p}<$ pos $p_{0}$ ), then new positive crossings $p_{i}$ on $\alpha_{i}$ occur as $p_{-m_{3}} \cdots$ $p_{-1} p_{0} p_{1} \cdots p_{m_{4}}$ in $W\left(f_{1}, R_{1}\right)$. If $\operatorname{pos}\left(\bar{p}_{0}\right)<\operatorname{pos}\left(p_{0}\right)$, then $m_{1} \leqslant m_{4}$ and $m_{2} \leqslant m_{3}$.

Suppose now that $P_{0}=\left(\bar{p}_{0}, p_{0}\right)$ is a pair in $P_{0}=P-\{P, Q\}$ and pos $\bar{p}$ $<\operatorname{pos} \bar{p}_{0}$. Because pos $\bar{p}_{0}<\operatorname{pos} p_{0}$, it follows that pos $\bar{p}_{i}<\operatorname{pos} p_{i}$. Hence $P_{i}=\left(\bar{p}_{i}, p_{i}\right)$ is a pair and will be included in $P_{1}$. Schematically, these new pairs and their positions in $W\left(f_{1}, R_{1}\right)$ can be described as follows:

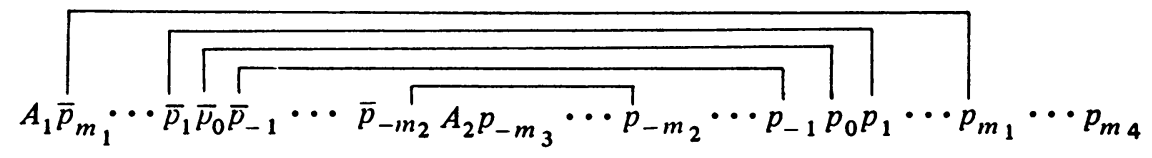
where $A_{1}$ and $A_{2}$ are subwords. From this configuration it is evident that each $P_{i}$ is similar to $P_{0}$.

The arrangement of new crossings near $\beta$ is analogous and new pairs are chosen as above. To complete the pairing $P_{1}$ on $W\left(f_{1}, R_{1}\right)$, also include all pairs in $P_{0}$. Define $S\left(P_{i}\right)=P_{0}, S\left(Q_{i}\right)=Q_{0}$ and $S\left(R_{0}\right)=R_{0}$ if $R_{0} \in P_{0}$.

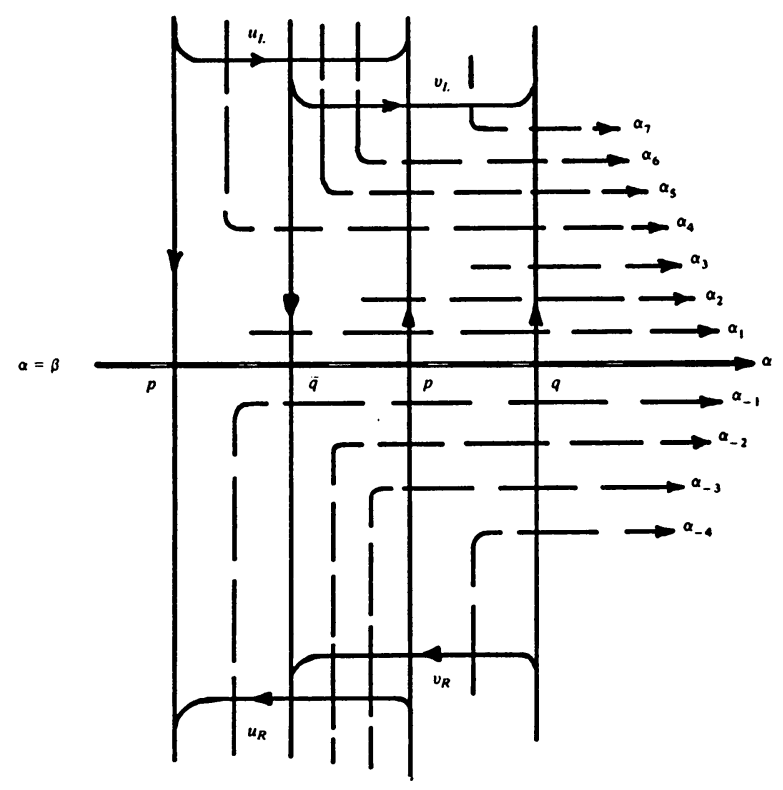

FIGURE 5

3. Reflections and linking. Let $W(f, R)$ be a crossing word for $f$ and assume that $(P, \wedge)$ is a pairing on $W(f, R)$. Let $P$ and $Q$ be linked pairs in $P$ $(\wedge(P, Q)=1)$. Then the normal loop $f_{1}=\bmod (f, P, Q)$ can be constructed and the reflection $W(P, Q)$ can be formed. Then $W(P, Q)$ induces a linking relation $\Lambda_{1}$ on $P_{0}=P-\{P, Q\}$. By Proposition 2.4 , a raying $R_{1}$ for $f_{1}$ and pairing $P_{1}$ on $W\left(f_{1}, R_{1}\right)$ can be chosen so that $P_{0} \subseteq P_{1}$. Moreover, each pair $R_{1}$ in $P_{1}$ is similar to a pair $S\left(R_{1}\right)$ in $P_{0}$ with respect to the linking induced by $W\left(f_{1}, R_{1}\right)$. Consequently, the linking of pairings on words obtained by mod- 
ifications can be studied combinatorially by looking only at the corresponding reflections and this section is concerned with that study. In particular, it will be shown how $\Lambda_{1}$ can be derived from $\Lambda$. Also, some consequences of similarity will be examined.

If $R \in P$, then $R$ and $\wedge$ determine a function $\wedge(R,-): P \rightarrow\{0,1\}$ where $\wedge(R,-)(S)=\bigwedge(R, S)$.

3.1. Proposition. Assume that $\wedge(P, Q)=1$ and $\Lambda_{1}$ is the relation on $P_{0}=P-\{P, Q\}$ induced by the reflection $W(P, Q)$. Then the following conditions are equivalent for any pairs $R$ and $S$ in $P_{0}$.

(1) $\bigwedge_{1}(R, S) \neq \wedge(R, S)$.

(2) The functions $\wedge(R,-)$ and $\Lambda(S,-)$ are neither equal nor identically zero on $\{P, Q\}$. For example, $\wedge(R, P)=\wedge(S, Q)=1$ but $\wedge(R, Q)=$ 0 is such an instance.

(3) The pair $R$ and the pair $S$ each intersect two of the four subwords of $W(f, R)$ determined by $P$ and $Q$ and exactly one subword does not intersect $R \cup S$.

If any of these conditions holds, we say that $(R, S)$ depends on $(P, Q)$.

PROOF. Since linking properties of a pair depend only on the location of the crossings in the word, both crossings of a pair will be denoted by the same letter. Let $W(f, R)=A p B q C p D q$. Then $W(P, Q)=A D C B$. Since in any of the conditions (1), (2), and (3) $R$ can be exchanged with $S$ or $P$ with $Q$, only representative cases will be treated.

(3) $\Rightarrow$ (1) Assume that $A$ is the subword containing one letter from each pair $R$ and $S$. Then since the other two letters are in different subwords $B$, $C$, or $D$, the transformation $A B C D \rightarrow A D C B$ transposes these letters. Hence $\bigwedge_{1}(R, S) \neq \wedge(R, S)$.

(1) $\Rightarrow$ (3) If $R$ intersects only one subword, then since the order within a subword is unaffected, $\bigwedge_{1}(R, S)=\bigwedge(R, S)$. Thus both $R$ and $S$ intersect two different subwords. If only two subwords are intersected then $\bigwedge_{1}(R, S)=$ 1 and $\Lambda(R, S)=1$ if and only if the letters $r$ and $s$ occur in the same order in both subwords. Assume all four subwords are intersected and $s$ is in $A$. Then $\bigwedge_{1}(R, S)=0$ and $\Lambda(R, S)=0$ if and only if the letters of $R$ occur in adjacent subwords. Thus $R$ and $S$ must intersect exactly three subwords.

(2) $\Rightarrow$ (3) The following typical case will be considered. Assume that $\bigwedge(R, P)=\bigwedge(S, Q)=1$ and $\Lambda(R, Q)=0 . \quad$ From $\wedge(R, P)=1$ and $\Lambda(R, Q)=0$ it follows that $R$ intersects two adjacent subwords separated by $p$. Since $\wedge(S, Q)$ $=1, S$ must intersect one of these subwords but not the other, so a total of three subwords is intersected.

(3) $\Rightarrow$ (2) Assume that $R$ intersects both $A$ and $B$ and $S$ intersects $A$ 
and $C$. Then $\bigwedge(R, P)=\bigwedge(S, Q)=1$ and $\bigwedge(R, Q)=0$. Other cases are essentially the same.

3.2. COROLlary . (1) Similarity is preserved under reflections.

(2) Dependence is preserved by similarity.

PRoof. (1) Assume that $R$ and $R^{\prime}$ are similar with respect to $\Lambda$. From $3.1(2),(R, S)$ depends on $(P, Q)$ iff $\left(R^{\prime}, S\right)$ depends on $(P, Q)$. Thus $\bigwedge(R, S)=\bigwedge\left(R^{\prime}, S\right)$ implies that $\bigwedge_{1}(R, S)=\bigwedge_{1}\left(R^{\prime}, S\right)$.

(2) Dependence, as characterized in $3.1(2)$, is determined by $\Lambda$.

3.3. Lemma. If $P \wedge P^{\prime}$, then $P^{\prime}$ does not link any other pair with respect to $\bigwedge_{1}$ or any subsequent reflection.

Proof. From $P \wedge P^{\prime}$ it follows that $\wedge\left(P^{\prime}, Q\right)=1$ and $\wedge\left(P^{\prime}, P\right)=0$. Let $R \in P_{0}=P-\{P, Q\}$ and assume $\Lambda\left(P^{\prime}, R\right)=1$. Then $\Lambda(P, R)=1$ so by $3.1(2),\left(P^{\prime}, R\right)$ depends on $(P, Q)$. Hence $\bigwedge_{1}\left(P^{\prime}, R\right)=0$. If $\Lambda\left(P^{\prime}, R\right)$ $=0$, then $\Lambda(P, R)=0$ so $\left(P^{\prime}, R\right)$ does not depend on $(P, Q)$. Thus $\bigwedge_{1}\left(P^{\prime},-\right)$ is identically zero on $P_{0}$. By characterization, $3.1(2),\left(P^{\prime}, R\right)$ is not dependent on any pair of pairs and hence $\Lambda_{2}\left(P^{\prime},-\right)$ is also identically zero.

3.4. Lemma. Let $P \wedge P^{\prime}$ and $Q \wedge Q^{\prime}$. Then $W(P, Q)$ and $W\left(P^{\prime}, Q^{\prime}\right)$ induce the same linking relation on $P-\left\{P, Q, P^{\prime}, Q^{\prime}\right\}$.

Proof. Dependence, as characterized by 3.1 (2), is preserved under similar-

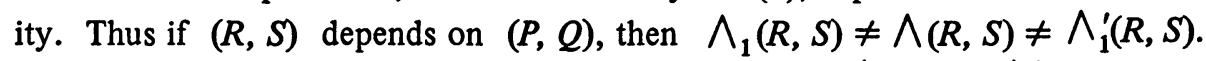
If $(R, S)$ does not depend on $(P, Q)$, then $\bigwedge_{1}(R, S)=\bigwedge(R, S)=\bigwedge_{1}^{\prime}(R, S)$.

3.5. Proposition. Let $C=\left(P_{1}, \cdots, P_{2 m}\right)$ and $C^{\prime}=\left(P_{1}^{\prime}, \cdots, P_{2 m}^{\prime}\right)$ be sequences in $P$ such that $P_{i} \wedge P_{i}^{\prime}$ for $1 \leqslant i \leqslant 2 m$. Then $C$ is a decomposition sequence $(\S 1)$ for $P$ if and only if $C^{\prime}$ is.

Proof. Induction on $m$ will be used. When $m=0, C=C^{\prime}=\varnothing$ so both are decomposition sequences if and only if $P$ is properly nested (no pairs link). Let $m>0$ and assume that $C$ is a decomposition sequence. Then $\left(P_{3}, \cdots, P_{2 m}\right)$ is a decomposition sequence for $P^{\prime}=P-\left\{P_{1}, P_{2}, P_{1}^{\prime}, P_{2}^{\prime}\right\}$ with respect to the relation $\bigwedge_{1}$ induced by $W\left(P_{1}, P_{2}\right)$. By $3.2(1), P_{i} \wedge P_{i}^{\prime}$ implies that $P_{i} \wedge_{1} P_{i}^{\prime}$ for $3<i<2 m$. Thus, by induction, $\left(P_{3}^{\prime}, \cdots, P_{2 m}^{\prime}\right)$ is a decomposition sequence for $\left(P^{\prime}, \Lambda_{1}\right)$. But from 3.4 it follows that $\Lambda_{1}=\Lambda_{1}^{\prime}$ on $P^{\prime}$. Thus $\Lambda_{m}^{\prime}$ is identically zero on $P^{\prime}-\left\{P_{3}^{\prime}, \cdots, P_{2 m}^{\prime}\right\}=P-\left(C^{\prime} \cup\left\{P_{1}, P_{2}\right\}\right)$. By 3.3, $\Lambda_{m}^{\prime}\left(P_{1},-\right)$ and $\Lambda_{m}^{\prime}\left(P_{2},-\right)$ are zero so $\Lambda_{m}^{\prime}$ is zero on $P-C^{\prime}$.

3.6. Proposition. Assume that $S: P_{1} \rightarrow P_{0} \subseteq P_{1}$ is a projection such that $S\left(R_{1}\right) \wedge R_{1}$ for each $R_{1} \in P_{1}$. Let $C$ be a decomposition sequence for $P_{0}$. Then $C$ is also a decomposition sequence for $P_{1}$. 
PRoof. Let $C=\left(P_{1}, \cdots, P_{2 m-2}\right)$. Assume that $R \in P_{1}-C$ and let $R_{0}=S(R)$. If $R_{0} \in C$, then, by $3.3, R$ does not link with respect to $\wedge_{m}$. Suppose that $R_{0} \notin C$. $P_{0}-C$ is properly nested with respect to $\Lambda_{m}$. But similarity is preserved under reflections so $R_{0}$ and hence $R$ can link no pair in $P_{1}-C$ with respect to $\Lambda_{m}$.

4. Pairings induced by extension of $f$. Throughout this section $F: T_{n} \rightarrow$ $E$ is an immersion which extends $f$ and $R$ is a raying for $f$. An immediate consequence, due to Gramain [8, Theorem $2^{\prime}$, p. 364], is that the tangent winding number of $f$ is $1-2 n$. Paralleling Marx [12] it will be shown how $F$ determines a unique pairing $P_{F}$ on $W(f, R)$ and it will be proved that $P_{F}$ is a $T_{n}$ assemblage. Further, $F$ and any decomposition sequence of length $2 n$ in $P_{F}$ defines a representation $(\S 1)$ of $T_{n}$ as a disk with $2 n$ strips attached. The propositions proved in 4.1-4.4 also hold when $F$ is properly interior as long as branch points are not mapped onto the rays. The following arc lifting lemma specializes Marx's formulation for interior extensions [Marx, 10, Theorem 1, p. 49].

4.1. Lemma. Let $\alpha:[0,1] \rightarrow E$ be a simple arc from $a$ to $b$ with $F(x)=a$. Assume that $\alpha$ and $f$ are transverse, and if $x \in S$, then $[\alpha]$ is to the left of $[f]$ at $a$.

Then there is a unique simple arc $X$ in $T_{n}$ from $x$ to $y$ such that

(1) $F \mid X$ is a diffeomorphism into $[\alpha]$,

(2) $y \notin S$ implies that $F(y)=b$,

(3) $X \cap S \subseteq\{x, y\}$.

4.2. PRoposition. Let $\bar{p}$ be a negative crossing on the ray $\alpha \in R$. Then there is a unique arc $X$ in $T_{n}$ from $\bar{x}$ to $x$ and a positive crossing $p$ on $\alpha$ such that

(1) $P=(\bar{p}, p)$ is a pair-i.e., $\operatorname{pos}(\bar{p})<\operatorname{pos} p$,

(2) $X \cap S=\{\bar{x}, x\}$,

(3) F maps $X$ diffeomorphically onto $\alpha_{P}$, the closed segment of $\alpha$ from $\bar{p}$ to $p$.

Proof. Because $\alpha$ and $f$ are transverse, $\bar{p}$ is not a node and hence has only one preimage $\bar{x}$ on $S$. By 4.1, there is a unique lift $X$ from $\bar{x}$ to $x$ of the segment of $\alpha$ beginning at $p$. If $x \notin S$, then 4.1 implies that $F(x)=$ $\alpha(1)$ (the endpoint of $\alpha$ ). But $\alpha(1) \in E_{\infty}$, the unbounded component, so $\alpha(1)$ $\notin F\left(T_{n}\right)$. Hence $x \in S$ and $f(x)=F(x)=p$ is a crossing on $\alpha$. Because $F$ is sense preserving, $\operatorname{sgn}(p)=1$ and $\operatorname{pos}(\bar{p})<\operatorname{pos}(p)$.

4.3. Definition. In $4.2, F$ determines a pair $P=(\bar{p}, p)$ for each negative crossing $\bar{p}$. Let $P_{F}$ be the collection of all such pairs. It will be shown in 
4.4, to follow, that these pairs are disjoint and hence $P_{F}$ is a pairing. The arc $X$ in 4.2 will be called the chord which lifts the pair $P$ and will also be denoted by $X_{P}^{F}$ or $X_{P}$.

4.4. Lemma. Let $P, Q \in P_{F}$ and assume that $P \neq Q$. Then $P$ and $Q$ are disjoint.

PROof. It suffices to show that $X_{P} \cap X_{Q}=\varnothing$. Assume the crossings in $P$ and $Q$ are on rays $\alpha$ and $\beta$, respectively. If $\alpha \neq \beta$, then $\alpha$ and $\beta$ are disjoint so $X_{P} \cap X_{Q}=\varnothing$. If $\alpha=\beta$ and $X_{P} \cap X_{Q} \neq \varnothing$, then $y \in X_{P} \cap X_{Q}$ can be chosen so that $F(y)$ is not a crossing on $\alpha$. By 4.1, there is a unique lift $X_{1}$ beginning at $y$ of $\left(F\left(x_{1}\right), \alpha(1)\right) \alpha$ and a unique lift $X_{2}$ of $(F(x), \alpha(0))(-\alpha)$ beginning at $y$. But then $X_{P}=-X_{2}+X_{1}=X_{Q}$, so $P=Q$.

The next proposition serves as the induction step in the proof of 4.6 and in the proof that the pairing $P_{F}$ is a $T_{n}$-assemblage.

4.5. Proposition. Assume that $P, Q \in P_{F}$ and $P$ links $Q$. Then there are tubular neighborhoods $N_{P}$ of $X_{P}$ and $N_{Q}$ of $X_{Q}$ such that $T_{n}$ $\left(N_{P} \cup N_{Q}\right)$ is diffeomorphic to $T_{n-1}$. Also, $F \mid T_{n-1}$ extends $f_{1}=$ $\bmod (f, P, Q)$.

4.6. Corollary. Assume that $\left(P_{1}, \cdots, P_{2 n}\right)$ is a decomposition sequence for $P_{F}$. Then there are tubular neighborhoods $N_{i}$ of $X_{P_{i}}$ such that $T_{n}-\bigcup_{i=1}^{2 n} N_{i}$ is a closed disc. Moreover, the reattaching of the neighborhoods $N_{i}$ to this disc corresponds to the process described in $\S 1$ to obtain representations of $T_{n}$. (The antipodal representation corresponds to the case when $P_{i}$ links $P_{j}$ for $i \neq j, 1 \leqslant i, j \leqslant 2 n$.)

The next lemma will be used in 4.8 to obtain immersions of $T_{n}$ from immersions of $T_{n-1}$ by extending $F$ over attached strips. This lemma can also be used to smooth the union of two mappings whose domains intersect properly.

4.7. Lemma. Let $M \cup D$ be an oriented surface where $D$ is a closed disc such that

(1) $D \cap M$ is the disjoint union of a finite number of closed discs;

(2) $\gamma: \partial D \rightarrow E$ is an embedding (one-to-one immersion). Then if $G: M \rightarrow E$ is an immersion which agrees with $\gamma$ on $\partial D \cap M$, there is an immersion $\bar{G}: M \cup D \rightarrow E$ such that $\bar{G}|D=\gamma, \bar{G}|(M-D)=G \mid(M-D)$, and $\bar{G} \mid D$ is a diffeomorphism.

Proof. Let $V$ be a normal unit vector field on $\partial D$ such that if $x \in M$, then $V(x)$ is tangent to $M$ at $x$. Assume also that $V$ is to the right of $\partial D$ 
and let $V_{G}$ be the composition of $V \mid(\partial D \cap M)$ with the differential $d G$ of $G$. Because $\partial D \cap M$ consists of a finite number of closed disjoint arcs, $V_{G}$ can be extended to all of $\partial D$. By Lemma 7 of [Francis, 2], there is a $C^{1}$ diffeomorphism $H: D \rightarrow E$ such that $H \mid \partial D=\gamma$ and $d H \circ V=V_{G}$. Then $\bar{G}=$ $G \mid(M-D)+H$ is $C^{1}$.

4.8. Proposition. Assume that $P$ and $Q$ are linked pairs from $W(f, R)$ and $f_{1}=\bmod (f, P, Q)$. Assume that $F_{1}: T_{n-1} \rightarrow E$ is an immersion which extends $f_{1}$ and let $P_{0}$ be the collection of pairs determined by $F_{1}$ and the negative letters in $W(P, Q)$. Then there is an immersion $F: T_{n} \rightarrow E$ which extends $f$ and is such that $P_{F}=P_{0} \cup\{P, Q\}$.

Proof. Refer again to Figure 4. Attach opposite ends of a rectangular strip $D_{1}$ to the segments of $\partial T_{n-1}$ which are mapped onto $u_{L}$ and $u_{R}$ (Figure 6). Similarly, attach a strip $D_{2}$ at $f_{1}^{-1}\left(v_{L}\right)$

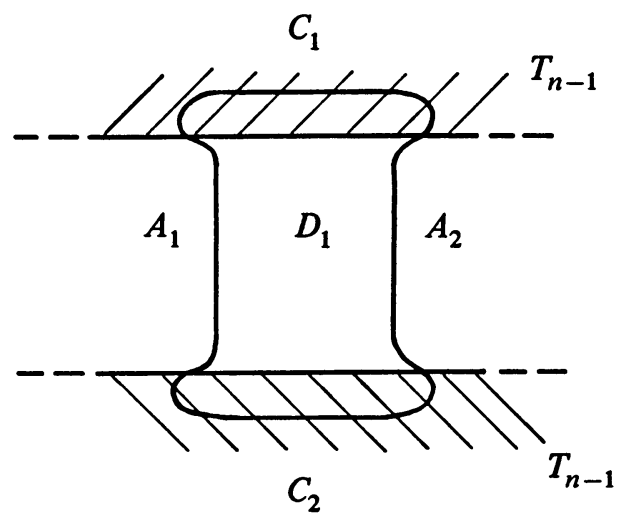

FIGURE 6

and $f_{1}^{-1}\left(v_{R}\right)$. Because $P$ and $Q$ are linked, the boundary of the resulting space is connected and so a copy of $T_{n}$ is obtained.

Now choose simple arcs $C_{1}$ and $C_{2}$ in $T_{n-1}$ (see Figure 6) so that if $A_{1}$ and $A_{2}$ denote the unattached segments of $\partial D_{1}$, then $C_{1} \cup C_{2} \cup A_{1} \cup$ $A_{2}$ is a smooth loop which bounds a disc in $T_{n}$. Require also that $C_{1}$ and $C_{2}$ do not intersect any chords which lift pairs in $P_{0}$. Let $\gamma=F_{1} \mid\left(C_{1} \cup C_{2}\right) \cup$ $\left(f \mid A_{1} \cup A_{2}\right)$. Then $F_{1}$ can be extended by 4.7 to $\bar{F}_{1}: T_{n-1} \cup D_{1} \rightarrow E$. Extend $F_{1}$ to $T_{n-1} \cup D_{1} \cup D_{2}$ by a similar application of 4.7 to obtain $F: T_{n} \rightarrow E$. Since $F \mid D_{1} \cup D_{2}$ is a diffeomorphism, $P$ and $Q \in P_{F}$. Also, $F$ agrees with $F_{1}$ on all chords which lift pairs in $P_{0}$. Thus $P_{F}=P_{0} \cup\{P, Q\}$.

5. Proof of Theorem I. This section consists of the proofs of the two parts of Theorem I. These propositions establish correspondences between exten- 
sions of normal loops and assemblages. For both parts, assume that $f: S \rightarrow E$ is a normal loop with raying $R$.

5.1. Proposition. Let $F: T_{n} \rightarrow E$ be an immersion which extends $f$. Then $\tau(f)=1-2 n$ and the pairing $P_{F}$ on $W(f, R)$ determined by $F$ is a $T_{n}$-assemblage.

PROof. By Gramain [7, p. 364], $\tau(f)=1-2 n$. Induction on $n$ will be used to show that $P_{F}$ (as obtained in 4.3) is a $T_{n}$-assemblage (1.1). The case for $n=0$ was proved by Blank [1] and Marx [12], so assume that $n>0$. If $P_{F}$ were properly nested, then $\tau(f)$ would be greater than or equal to 1 . Hence linked pairs $P$ and $Q$ in $P_{F}$ can be chosen. By 4.5, there are neighborhoods $N_{P}$ of $X_{P}$ and $N_{Q}$ of $X_{Q}$ such that $T_{n}-\left(N_{p} \cup N_{q}\right)=T_{n-1}$ and $F_{1}=F \mid T_{n}-\left(N_{P} \cup N_{Q}\right)$ extends $f_{1}=\bmod (f, P, Q)$. Let $R_{1}$ be a raying for $f_{1}$ constructed as in 2.4. Let $P_{1}$ be the pairing on $W\left(f_{1}, R_{1}\right)$ defined as in 2.4 , so that $S\left(R_{1}\right) \wedge_{1} R_{1}$ for each $R_{1} \in P_{1}$. The rays in $R_{1}$ can be chosen sufficiently close to $\alpha$ and $\beta$ so that $F$ lifts a new pair $R_{1}$ if and only if $F$ lifts $S\left(R_{1}\right)$. Thus $P_{F_{1}}=$ $P_{1}$. By induction, $P_{1}$ is a $T_{n-1}$-assemblage and therefore $P_{1}$ contains a decomposition sequence $C^{\prime}=\left(P_{1}^{\prime}, \cdots, P_{2 n-2}^{\prime}\right)$. For $1 \leqslant i \leqslant 2 n-2$, let $P_{i}=$ $S\left(P_{i}^{\prime}\right)$. Then by $3.5, C=\left(P_{1}, \cdots P_{2 n-2}\right)$ is a decomposition sequence for $P_{1}$. Hence $P_{1}-C$ is properly nested with respect to $\wedge_{n}$. But $P_{F}-\{P, Q\} \subseteq P_{1}$ so $P_{F}-\left\{P, Q, P_{1}, \cdots, P_{2 n-2}\right\}$ is properly nested with respect to $\Lambda_{n}$. Therefore $\left(P, Q, P_{1}, \cdots, P_{2 n-2}\right)$ is a decomposition sequence of length $2 n$ and so $P_{F}$ is a $T_{n}$-assemblage.

5.2. Proposition. Assume that $P$ is a $T_{n}$-assemblage on $W(f, R)$ and $\tau(f)=1-2 n$. Then there is an immersion $F: T_{n} \rightarrow E$ which extends $f$ and $P_{F}=P$.

PROof. Induction on $n$ will be used. The case for $n=0$ was treated by Blank [1] and Marx [12] so assume $n>0$. Let $\left(P_{1}, \cdots, P_{2 n}\right)$ be a decomposition sequence in $P$. Let $f_{1}=\bmod \left(f, P_{1}, P_{2}\right)$. Form the raying $R_{1}$ and pairing $P_{1}$ for $f_{1}$ as in 2.4. Then if $P_{0}=P-\left\{P_{1}, P_{2}\right\}, P_{0} \subseteq P_{1}$ and each pair $R_{1}$ in $P_{1}$ is similar to a pair $S\left(R_{1}\right)$ in $P_{0}$. Because $P_{0} \subseteq P_{1},\left(P_{3}, \cdots, P_{2 n}\right)$ is a decomposition sequence for $P_{1}$. From $2.2, \tau\left(f_{1}\right)=\tau(f)+2=1-2(n-1)$. Thus by induction, there is an immersion $F_{1}: T_{n-1} \rightarrow E$ such that $F_{1}$ extends $f$, and $P_{F_{1}}=P_{1}$. Hence $P_{0} \subseteq P_{F_{1}}$ so by 4.8 , there is an immersion $F: T_{n} \rightarrow E$ which extends $f$ and $P_{F}=P_{0} \cup\left\{P_{1}, P_{2}\right\}=P$.

6. Equivalence. As defined in $\S 1$, two immersions $F, G: T_{n} \rightarrow E$ which extend a normal loop $f$ are equivalent if there is an orientation preserving 
diffeomorphism $h: T_{n} \rightarrow T_{n}$ such that $F=G \circ h$. Theorem II, which shows that this equivalence is determined by equality of assemblages, will be proved in this section. Because a reparametrization of $S$ can be extended to an orientation preserving diffeomorphism of $T_{n}$ (6.2), the existence and equivalence of extensions of normal loops depend only on the image curve. It also follows that these questions are independent of the diffeomorphic copy of $T_{n}$ used. Thus $T_{n}$-assemblages determine extensions up to orientation preserving diffeomorphisms of $T_{n}$. Francis [6] has produced an example of a normal curve with four $T_{0}$ assemblages. One of the induced extensions can be obtained from another of them by compositions with rotations of $T_{0}$ and $E$. Thus equality of assemblages will not classify extensions if diffeomorphisms of the plane are allowed. Two of the assemblages in this example are isomorphic. A problem for later study will be to investigate equivalence determined by isomorphism of assemblages.

6.1. THEOREM II. Let $f: S \rightarrow E$ be a normal loop such that $\tau(f)=1-$ $2 n$ and let $R$ be a raying for $f$. Assume that $F, G: T_{n} \rightarrow E$ are immersions which extend $f$. Then $F$ and $G$ are equivalent iff $F$ and $G$ determine the same $T_{n}$-assemblage; i.e., $P_{F}=P_{G}$.

Proof. First, assume that $F$ and $G$ are equivalent. $P_{F}$ and $P_{G}$ are obtained as in 5.5. Let $\bar{p}$ be a negative crossing on a ray $\alpha \in R$. Then for some positive crossing $p$ on $\alpha, P=(\bar{p}, p) \in P_{F}$. Let $X_{P}^{F}$ be the unique chord from $\bar{x}$ to $x$ which lifts $P$ under $F$ and let $X=h\left(X_{P}^{F}\right)$ where $h: T_{n} \rightarrow T_{n}$ is a diffeomorphism such that $F=G \circ h$. Because $h$ is a diffeomorphism, $X \cap S=$ $\{h(\bar{x}), h(x)\}$. Since $G(X)=G \circ h\left(X_{P}^{F}\right)=F\left(X_{P}^{F}\right), G$ maps $X$ diffeomorphically onto the segment $\alpha_{P}$ of $\alpha$. From 5.4 it follows that $X=X_{P}^{G}$ and so $P \in P_{G}$. Exchanging the roles of $F$ and $G$ completes the argument.

Now assume that $P_{F}=P_{G}$. The proof is by induction on $n$. The case for $n=0$ was treated by Marx [13]. When $n>0, P$ contains linked pairs $P$ and $Q$. Let $N_{1}$ of $X_{P}^{F}, N_{2}$ of $X_{P}^{G}, M_{1}$ of $X_{Q}^{F}$, and $M_{2}$ of $X_{Q}^{G}$ be tubular neighborhoods such that $T_{n}-\left(N_{i} \cup M_{i}\right)=T_{n-1}, i=1,2$, as in 4.7. Then $F_{1}$ $=\left.F\right|_{T_{n-1}}$ and $G_{1}=\left.G\right|_{T_{n-1}}$ extend $f_{1}=\bmod (f, P, Q)$. Choose a raying $R_{1}$ for $f_{1}$ and pairing $P_{1}$ as in 2.4 so that a new pair $R_{1} \in P_{1}$ is lifted by $F$ and $G$ if and only if the corresponding pair $S\left(R_{1}\right)$ in $P_{0}$ is lifted. Then $P_{F_{1}}=P_{G_{1}}$. By induction, there is a diffeomorphism $h_{1}: T_{n-1} \rightarrow T_{n-1}$ such that $F_{1}=G_{1} \circ h_{1}$. If

$$
h=h_{1}+\left[\left(G \mid N_{2} \cup M_{2}\right)^{-1} \circ\left(F \mid N_{1} \cup M_{1}\right)\right],
$$

then $F=G \circ h$.

6.2. Lemma. Assume that $f, g: S \rightarrow E$ are normal loops such that $[f]$ $=[g]$. Then there is a diffeomorphism $\eta: T_{n} \rightarrow T_{n}$ such that $f=g \circ(\eta \mid S)$. 
PROOF. Partition $S$ into intervals $\left[t_{0}, t_{1}\right], \cdots,\left[t_{k-1}, t_{k}\right]$ with $t_{0}=t_{k}$ such that $f_{i}=f \mid\left[t_{i-1}, t_{i}\right]$ is one-one. Then, for each $i, 1 \leqslant i \leqslant k$, there is a unique interval $\left[s_{i-1}, s_{i}\right] \subseteq S$ which is mapped diffeomorphically onto $\left[f_{i}\right]$ by $g$. Let $g \mid\left[s_{i-1}, s_{i}\right]=g_{i}$. Define $\eta_{0}$ on $\left[t_{i-1}, t_{i}\right]$ by $\eta_{0}(t)=g_{i}^{-1} \circ f_{i}(t), 1 \leqslant i \leqslant k$. Then $f=g \circ \eta_{0}$. Extend $\eta_{0}$ to an annulus $A_{1} \subseteq T_{n}$ which contains $S$ and let $A_{2} \supsetneq A_{1}$ be an annulus in $T_{n}$. Then a diffeomorphism $\eta: T_{n} \rightarrow T_{n}$ can be defined so that $\eta \mid T_{n}-A_{2}=1_{T_{n}-A_{2}}$ and $\eta \mid A_{1}=\eta_{0}$.

Proposition. Assume that $F, G: T_{n} \rightarrow E$ extend normal loops fand $g$ and $[f]=[g]$. Let $R$ be a raying for $f$ and $g$. Then $F$ and $G$ are equivalent if and only if $P_{F}=P_{G}$.

Proof. Assume that there is a diffeomorphism $h: T_{n} \rightarrow T_{n}$ such that $F G \circ h$. That $P_{F}=P_{G}$ follows as in 6.1 .

Next, assume that $P_{F}=P_{G}$ and let $\eta: T_{n} \rightarrow T_{n}$ be the diffeomorphism obtained in 6.2. Then $G \circ \eta$ and $F$ both extend $f$. By part one above, $P_{G}=P_{G \circ \eta}$. Thus 6.1 implies that $F$ and $G \circ \eta$ are equivalent and hence $F$ and $G$ are equivalent.

7. Applications. In this section a formulation of a $T_{1}$-assemblage will be given which does not require the reflection operation. It will be used to determine, by way of an example, three inequivalent extensions of a particular loop to $T_{1}$. A result of Marx [12, Theorem 6, p. 13] will be used to show that if a normal loop $f$ has a $T_{n}$ assemblage, then $f$ can be extended to an interior map $F: T_{n} \rightarrow E$. Also, for each $n$, a normal loop $f_{n}$ will be described which has exactly one class of extensions to $T_{n}$. These loops are canonical in that among extendible loops they have the minimum number of nodes (self-intersections) and for each $n$, an extendible loop with the minimum number of nodes is equivalent to $f_{n}$.

7.1. Theorem. Let $P$ be a pairing on $W(f, R)$. Then $P$ is a $T_{1}$-assemblage if and only if there is a partition $P=N \cup L_{1} \cup L_{2} \cup L_{3}$ such that (1) $L_{1} \times L_{2} \neq$ $\varnothing$ and (2) $\wedge(R, S)=1$ if and only if $R \in L_{i}, S \in L_{j}$, and $i \neq j$.

Proof. First, assume $P$ is a $T_{1}$-assemblage with decomposition sequence $(P, Q)$. Let

$$
\begin{aligned}
N & =\{R \in P: \wedge(R, P)=\bigwedge(R, Q)=0\}, \\
L_{1} & =\{R \in P: \wedge(R, P)=0 \text { and } \bigwedge(R, Q)=1\}, \\
L_{2} & =\{R \in P: \bigwedge(R, P)=1 \text { and } \bigwedge(R, Q)=0\}, \\
L_{3} & =\{R \in P: \wedge(R, P)=\bigwedge(R, Q)=1\} .
\end{aligned}
$$

Although $L_{3}$ may be empty, $P \in L_{1}$ and $Q \in L_{2}$. Let $R, S \in P$. In view of 3.1(2), $(R, S)$ depends on $(P, Q)$ if and only if $R \in L_{i}$ and $S \in L_{j}$ for $i \neq j$. 
Because $(P, Q)$ is a decomposition sequence, $\bigwedge_{1}(R, S)=0$ if $R, S \in P$ $-\{P, Q\}$. Thus $\bigwedge(R, S)=1$ if and only if $R \in L_{i}$ and $S \in L_{j}$ for $i \neq j$.

Now assume that $P$ is the union of $N, L_{1}, L_{2}$, and $L_{3}$, which satisfy (1) and (2) above. Then $P \in L_{1}$ and $Q \in L_{2}$ can be chosen so that $\wedge(P, Q)$ $=1$. To show that $(P, Q)$ is a decomposition sequence for $P$, assume that $R, S \in P-\{P, Q\}$. By $3.1(2),(R, S)$ depends on $(P, Q)$ if and only if $R \in L_{i}$ and $S \in L_{j}$ for $i \neq j$. Thus if $R \in L_{i}, S \in L_{j}$ and $i \neq j$, then $1=\wedge(R, S)$ $\neq \bigwedge_{1}(R, S)$. In all other cases, $\bigwedge_{1}(R, S)=\bigwedge(R, S)=0$.

7.2. ExAMPLE. Let $f$ be the following normal loop:

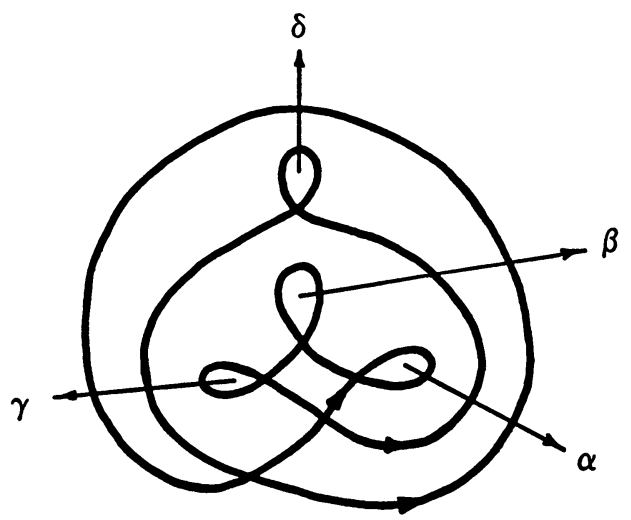

FIGURE 7

Every node of this curve is a cut point of the image [f]. Such curves have at most one class of extensions to the disc [Francis, 3]. In contrast, there are three $T_{1}$-assemblages for $W(f, R)$ and hence three classes of immersions of $T_{1}$ which extend $f$. Only the rays involved in the pairings are indicated and $\bar{p}_{k}$ denotes a negative crossing on a ray $p$ with $\operatorname{pos}\left(p_{k}\right)=k$.

$W$ (abbreviated): $\alpha_{3} \beta_{3} \delta_{2} \gamma_{3} \bar{\alpha}_{1} \bar{\beta}_{1} \bar{\gamma}_{1} \alpha_{2} \beta_{2} \bar{\delta}_{1} \gamma_{2}$
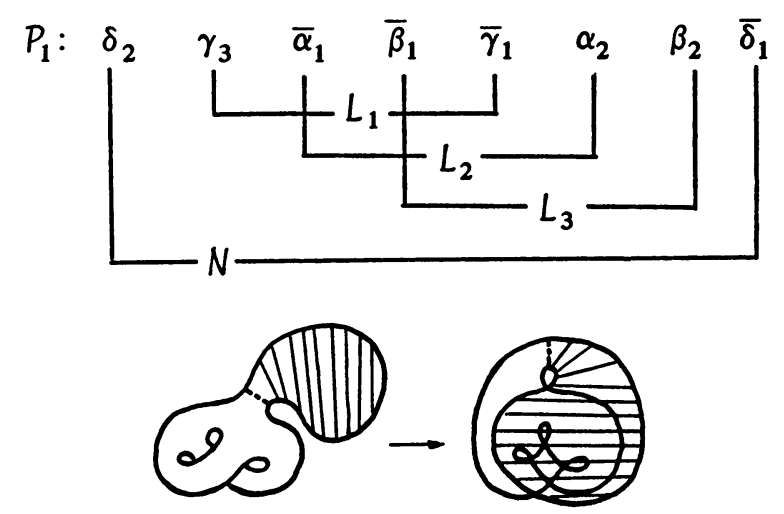

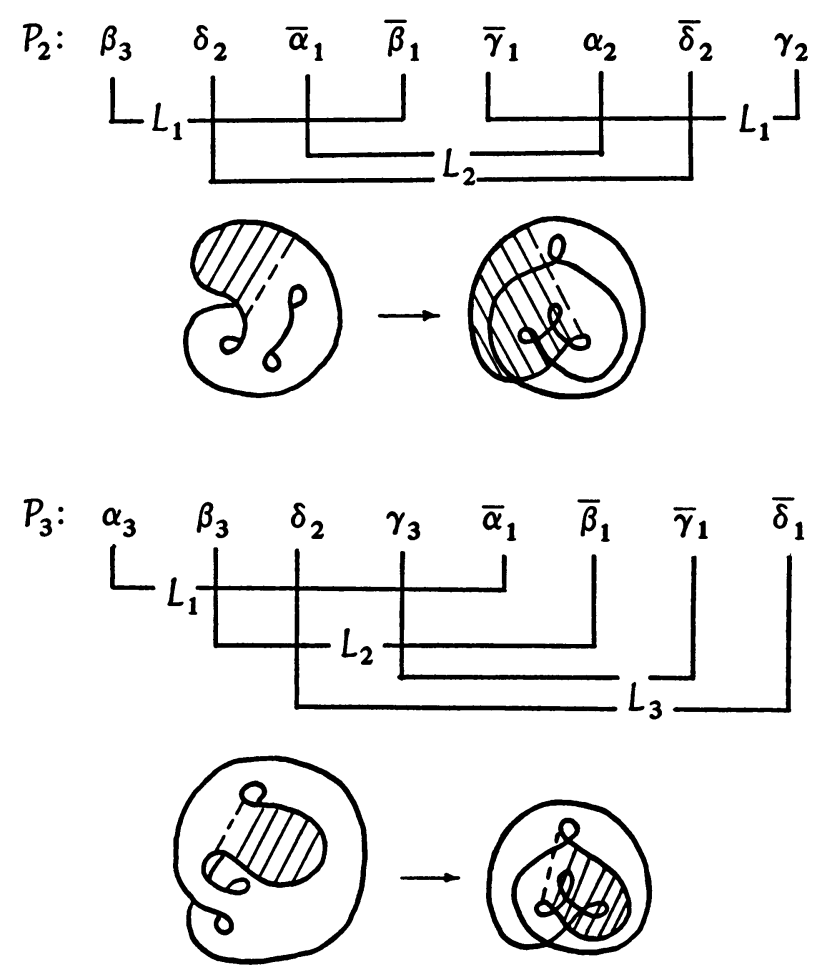

FIGURE 8

7.3. THEOREM. Let $f: S \rightarrow E$ be normal and assume that $R$ is a raying for $f$. If $P$ is a $T_{n}$-assemblage on $W(f, R)$, then there is an interior map $F: T_{n} \rightarrow E$ that extends $f$.

Proof. Let $C$ be a decomposition sequence of length $2 n$ in $P$ and let $f_{n}$ be the corresponding $n$th modification. Then by induction, 2.3, and 3.6 there is a properly nested pairing $P_{n}$ on $W\left(f_{n}, R_{n}\right)$. By a theorem of Marx $\left[12\right.$, Theorem 6], there is an interior map $F_{n}: T_{0} \rightarrow E$ that extends $f_{n}$. The process of extending a map to attached strips in the proof of 4.8 works equally well when the original map is properly interior. Thus an interior extension $F: T_{n} \rightarrow E$ of $f$ can be obtained. In terms of a representation of $T_{n}$ as in $\S 1, F$ is an immersion on the attached strips and interior on the disc.

7.4. Corollary. Let $f: S \rightarrow E$ be a normal loop. Then $f$ has nonnegative circulation if and only if for some $n$, there is an interior map $F: T_{n} \rightarrow$ $E$ which extends $f$.

Proof. Let $R$ be a raying for $f$. The winding number of $f$ about any point $x \in E-[f]$ is the sum of the signs of the crossings of $f$ and any ray from $x$ to $E_{\infty}$. Thus if $f$ has nonnegative circulation, a pairing exists for 
$W(f, R)$. But every pairing is a $T_{n}$-assemblage for some $n$ and hence by $7.3, f$ has an interior extension to $T_{n}$.

Conversely, if an interior extension exists, there is a corresponding pairing. Since each negative crossing is paired with a different positive crossing and at least one ray originates in each bounded component of $E-[f], f$ has nonnegative circulation.

7.5. CANONICAL LOOPS. Since normal loops with isomorphic intersection sequences are equivalent [Titus, 18 , Theorem 3, p. 49] the examples here will be specified by their intersection sequences. For each $n \geqslant 1$, let $f_{n}$ be a normal loop with intersection sequence

$$
\stackrel{+}{+}+\overrightarrow{1} 2233 \ldots(2 n \overline{+}+2)(2 n+2) \overline{1} \text {. }
$$

Thus $f_{n}$ has an initial positive node followed by $2 n$ simple negative loops. For $f_{2}$, see Figure 9 .
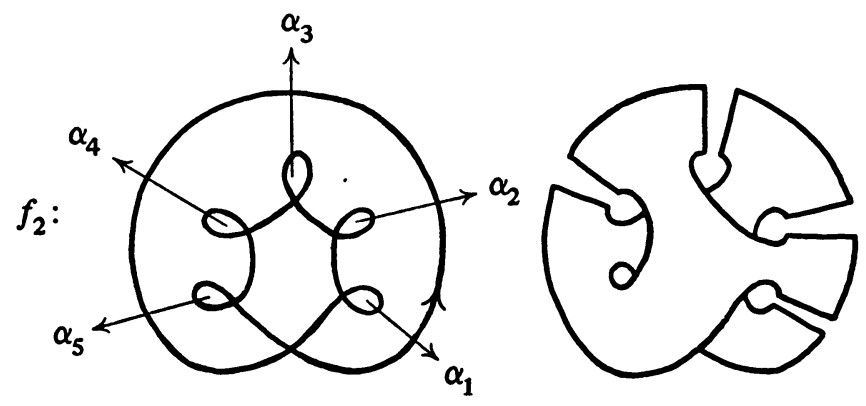

\section{FIGURE 9}

Again, only rays involved in pairings are indicated and letters are denoted as in 7.2 .

$W$ (abbreviated): $\left.(\alpha 1)_{2}(\alpha 2)_{2} \cdots(\alpha 2 n)_{2}(\alpha 2 n+1)_{2}(\overline{\alpha 1})_{1} \cdots \overline{(\alpha 2 n+1}\right)_{1}$.

Let $\left.P_{k}=(\overline{(\alpha k})_{1},(\alpha k)_{2}\right)$ for $1 \leqslant k \leqslant 2 n+1$. Then

$P=\left\{P_{1}, \cdots, P_{2 n+1}\right\}$ is the only pairing on $W\left(f_{n}, R_{n}\right)$.

$P-\left\{P_{1}, \cdots, P_{2 n}\right\}=\left\{P_{2 n+1}\right\}$ so $P$ is a $T_{n}$-assemblage and determines an extension $F_{n}: T_{n} \rightarrow E$ of $f_{n}$.

That $F_{n}$ and the decomposition sequence $\left\{P_{1}, \cdots, P_{2 n}\right\}$ determine a representation of $T_{n}$ as in 4.6 is illustrated in Figure 9.

To prove that the loops $f_{n}$ are canonical, some additional definitions and formulas will be needed.

7.6. Definition. A loop $f: S \rightarrow E$ which is normal except for a finite number of corners that do not occur at nodes will be called piecewise normal. 
An outside starting point for $f$ is any nonintersection point $P_{0}=f\left(x_{0}\right)$ in the closure of $E_{\infty}$. Define the sign of $P_{0}$ by $\lambda\left(P_{0}\right)=1$ if $E_{\infty}$ lies to the right of [f] at $P_{0}$ and $\lambda\left(P_{0}\right)=-1$ otherwise. $P_{0}$ and the orientation of $S$ induce an order on the nodes (self-intersections) of $[f]$ and on the points of $S$. Let $N_{i}$ denote the $i$ th node and let $f^{-1}\left(N_{i}\right)=\left\{\bar{x}_{i}, x_{i}\right\}$ where $\bar{x}_{i}<x_{i}$. Pairs of preimages of nodes determine a linking relation on nodes as did preimages of pairs of crossings and the same terminology will be used. For example, $\bigwedge\left(N_{i}, N_{j}\right)=1$ if $N_{i}$ links $N_{j}$. The sign of a node $N_{i}$ is defined by

$$
\lambda\left(N_{i}\right)=\operatorname{sgn} \operatorname{det}\left[\begin{array}{l}
f^{\prime}\left(\bar{x}_{i}\right) \\
f^{\prime}\left(x_{i}\right)
\end{array}\right] .
$$

If $E$ is a set of nodes, denote the sum of their signs by $\lambda(E)$.

A set $E\left(N_{1}\right)$ of essential nodes [Francis, 2, p. 282] for $f$ can be defined inductively as follows.

(1) $N_{1} \in E\left(N_{1}\right)$.

(2) Assume $N_{k} \in E\left(N_{1}\right)$. Then $N_{j} \in E\left(N_{1}\right)$ if $j$ is the smallest integer $>k$ such that $\Lambda\left(N_{i}, N_{j}\right)=0$ for each $N_{i} \in E\left(N_{1}\right)$.

Thus if $N_{k} \in E\left(N_{1}\right)$, then include the next node after $N_{k}$ which links none of the previously chosen nodes.

The next proposition was also proved by Titus [17, Theorem 5, p. 1091] and Francis [2, p. 283] and gives a variation of Whitney's formula [21, p. 281] $\tau(f)=\lambda\left(P_{0}\right)+\lambda(N)$, where $N$ is the set of all nodes of $f$.

7.7. Proposition. Let $P_{0}$ be an outside starting point on a piecewise normal loop $f$ and let $N_{i}$ be the ith node encountered after $P_{0}$ as $[f]$ is traced. Then $\tau(f)=\lambda\left(P_{0}\right)+\lambda\left(E N_{1}\right)$.

Proof. Induction on $n$, the number of nodes of $f$, will be used. If $n=0$, then $[f]$ is a positively or negatively oriented simple loop so $\tau(f)=\lambda\left(P_{0}\right)$.

Let $n>0$ and define $f_{1}=f \mid\left[x_{1}, \bar{x}_{1}\right]$ and $f_{2}=f \mid\left[\bar{x}_{1}, x_{1}\right]$. Let $P_{10}$ $=P_{20}=N_{1}$. Then $\tau(f)=\tau\left(f_{1}\right)+\tau\left(f_{2}\right)+\tau_{1}+\tau_{2}$ where $\tau_{i}$ is the contribution from the corner at $P_{0}$ for $f_{i}, i=1,2$. But $\tau_{1}=-\tau_{2}$ so $\tau(f)=\tau\left(f_{1}\right)+$ $\tau\left(f_{2}\right)$. Examination of the four possible orientation arrangements of $[f]$ near $P_{0}$ shows that $\lambda\left(P_{0}\right)+\lambda\left(N_{1}\right)=\lambda\left(P_{10}\right)+\lambda\left(P_{20}\right)$. Because no node of $f$ that is a node of $f_{1}$ or $f_{2}$ can link $N_{1}$, it follows easily that $E\left(N_{i}\right)=\left\{N_{j} \in\right.$ $E\left(N_{1}\right): N_{j}$ is a node of $\left.f_{i}\right\}, i=1,2$. Thus by induction,

$$
\begin{aligned}
\tau\left(f_{1}\right)+\tau\left(f_{2}\right) & =\lambda\left(P_{10}\right)+\lambda\left(E N_{11}\right)+\lambda\left(P_{20}\right)+\lambda\left(E N_{21}\right) \\
& =\lambda\left(P_{0}\right)+\lambda\left(E N_{1}\right) .
\end{aligned}
$$

7.8. Definition. The preimages of nodes induce a containment relation on nodes given by $N_{j} \subset N_{i}$ if $x_{i}<x_{j}<\bar{x}_{j}<\bar{x}_{i}$. 
The containment relation on a properly nested set of nodes (e.g., on $E N_{1}$ ) corresponds to a tree graph (see [2, p. 288] for examples).

7.9. ThEOREM. Assume that $f: S \rightarrow E$ is a normal loop which can be extended to an immersion of $T_{n}$. Then

(1) the number of nodes of $f, v(f)$, is at least $2 n+2$.

(2) if $\nu(f)=2 n+2$, then $f$ is equivalent to the loop $f_{n}$ defined in 7.5.

Proof. (1) Let $N_{1}$ be the first node that occurs after an outside starting point $P_{0}$ on $[f]$. Because $f$ has positive circulation, $\lambda\left(P_{0}\right)=\lambda\left(N_{1}\right)=1$. But, $\tau(f)=1-2 n$ and so by $7.71-2 n=\lambda\left(P_{0}\right)+\lambda\left(E N_{1}\right)$. Thus $f$ must have at least $2 n+1$ negative nodes in $E\left(N_{1}\right)$.

(2) Assume that $\nu(f)=2 n+2$. As noted above, counting $N_{1}$, there are at least $2 n+2$ nodes in the properly nested set $E\left(N_{1}\right)$; the tree graph for $E\left(N_{1}\right)$ thus includes all of the nodes of $f$. For any node $N_{k}$ in such a graph, the winding number of one of the components of $E-[f]$ is $-\lambda\left(P_{0}\right)$ plus the sum of the signs along the (unique) path in the tree from $P_{0}$ to $N_{k}$. Since $f$ has positive circulation, the only possible configuration is

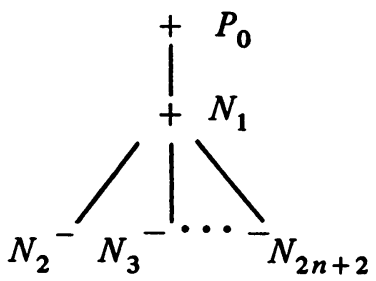

Therefore, $f$ and $f_{n}$ have isomorphic intersection sequences.

\section{REFERENCES}

1. S. J. Blank, Extending immersions of the circle, Dissertation, Brandeis University, 1967; Cf. V. Poenaru, Extensions des immersions en codimension 1 (d'après Blank), Séminaire Bourbaki: 1967/68, Exposé 342, Benjamin, New York, 1969. MR 41 \#3.

2. G. K. Francis, The folded ribbon theorem. A contribution to the study of immersed circles, Trans. Amer. Math. Soc. 141 (1969), 271-303. MR 39 \#463.

3. - Extensions to the disk of properly nested plane immersions of the circle, Michigan Math. J. 17 (1970), 377-383. MR 44 \#2209.

4. - Picard-Loewner problem, Geometry Seminar, University of Illinois, 1972.

5. - Spherical curves that bound immersed discs, Proc. Amer. Math. Soc. 41

(1973), 87-93.

6. - Branched and folded parametrizations of the sphere, Bull. Amer. Math. Soc. 80 (1973), $72-76$.

7. A. Gramain, Bounding immersions of codimension 1 in the Euclidean space, Bull. Amer. Math. Soc. 76 (1970), 361-365. MR 41 \#4560.

8. M. Heins, Complex function theory, Pure and Appl. Math., vol. 28, Academic Press, New York, 1968. MR 39 \#413.

9. J. Jewett, Differentiable approximations to light interior transformations, Duke Math. J. 23 (1956), 111-124. MR 18, 140. 
10. M. L. Marx, Normal curves arising from light open mappings of the annulus, Trans. Amer. Math. Soc. 120 (1965), 45-56. MR 33 \#3278.

11. - Light open mappings on a torus with a disc removed, Michigan Math. J. 15(1968), 449-456. MR 38 \#2750.

12. - Extensions of normal immersions of $S^{1}$ into $R^{2}$, Trans. Amer. Math. Scc. 187 (1974), 309-326.

13. M. L. Marx and R. F. Verhey, Interior and polynomial extensions of immersed circles, Proc. Amer. Math. Soc. 24 (1970), 41-49. MR 40 \#5879.

14. M. Morse and M. Heins, Topological methods in the theory of functions of a single complex variable. I. Deformation types of locally simple plane curves, Ann. of Math. (2) 46 (1945), 600-624. MR 8, 21.

15. - Deformation classes of meromorphic functions and their extensions to interior transformations, Acta Math. 79 (1947), 51-103. MR 8, 507.

16. S. Stoilow, Leçons sur les principes topologiques de la théorie des fonctions analytiques, Gauthier-Villars, Paris, 1938.

17. C. J. Titus, $A$ theory of normal curves and some applications, Pacific J. Math. 10 (1960), 1083-1096. MR 22 \#5014.

18. - The combinatorial topology of analytic functions on the boundary of a disk, Acta Math. 106 (1961) 45-64. MR 29 \#3652.

19. S. Troyer, Extending a boundary immersion to the disc with $n$ holes, Dissertation, Northeastern University, 1973.

20. J. H. C. Whitehead, Manifolds with transverse fields in euclidean space, Ann. of Math. (2) 73 (1961), 154-212. MR 23 \#A2225. 276-284.

21. H. Whitney, On regular closed curves in the plane, Compositio Math. 4 (1937),

22. G. T. Whyburn, Topological analysis, Princeton Math. Series, no. 23, Princeton Univ. Press, Princeton, N. J. , 1958; 2nd rev. ed., 1964. MR 20 \#6081; 29 \#2758.

\section{DEPARTMENT OF MATHEMATICS, UNIVERSITY OF ILLINOIS, URBANA, ILLINOIS 61801}

Current address: Computer Based Education Research Laboratory, University of Illinois, Urbana, Illinois, 61801 